M. Nakai

Nagoya Math. J.

Vol. 51 (1973), 131-135

\title{
DIRICHLET FINITE BIHARMONIC FUNCTIONS ON THE PLANE WITH DISTORTED METRICS
}

\author{
MITSURU NAKAI*
}

1. The Laplace-Beltrami operator $\Delta$ on a smooth manifold $M$ with a smooth Riemannian metric $d s^{2}=\sum_{i, j} g_{i j}(x) d x^{i} d x^{j}$ applied to a smooth function $\varphi$ takes the form $\Delta \varphi=g^{-1 / 2} \sum_{i, j}\left(g^{1 / 2} g^{i j} \varphi_{x^{j}}\right)_{x^{i}}$. Functions in the class $H^{2}(M)=\left\{u \in C^{4}(M) ; \Delta^{2} u=0\right\}$ are called biharmonic. The class $H(M)=H^{1}(M)=\left\{u \in C^{2}(M) ; \Delta u=0\right\}$ of harmonic functions is a subclass of $H^{2}(M)$. Let $D(M)$ be the class of functions $\varphi$ on $M$ having squareintegrable gradients, i.e. the Dirichlet integrals $D_{M}(\varphi)=\int_{M}|\operatorname{grad} \varphi|^{2} * 1$ are finite. In contrast with the harmonic null class $\mathcal{O}_{H D}=\{M ; H D(M)=\boldsymbol{R}\}$, $\boldsymbol{R}$ being the real number field (cf. Sario-Nakai [3]), we consider the biharmonic null class

$$
\mathcal{O}_{H^{2} D}=\left\{M ; H^{2} D(M)=H D(M)\right\} .
$$

This class was introduced and intensively studied by Nakai-Sario [1]. One of the main questions concerning the class (1) is: Does the property $M \in \mathcal{O}_{H^{2} D}$ have anything to do with the harmonic degeneracy of the ideal boundary of $M$ ?

Let $\boldsymbol{D}$ be the unit disk $|z|<1$ and $\boldsymbol{D}_{\alpha}$ be the disk $\boldsymbol{D}$ equipped with the Riemannian metric

$$
d s=(1-|z|)^{-\alpha}|d z|
$$

Nakai-Sario [1] proved

THEOREM 1. The manifold $\boldsymbol{D}_{\alpha}$ belongs to the null class $\mathcal{O}_{H^{2} D}$ if and only if $\alpha \geq 3 / 4$.

The case $\alpha=3 / 4$ was supplemented by O'Malla [2]. The significance of this assertion lies in an interesting contrast with the harmonic case:

Received November 5, 1971.

* Supported by Grant DA-ARO-D-31-124-71-G20, UCLA, Summer, 1971. 
$\boldsymbol{D}_{\alpha} \notin \mathcal{O}_{H D}$ for every $\alpha$. Let $\boldsymbol{C}$ be the finite plane $|z|<\infty$ and $\boldsymbol{C}_{\alpha}$ be the plane $C$ equipped with the Riemannian metric

$$
d s=(1+|z|)^{-\alpha}|d z|,
$$

a counter part of $D_{\alpha}$. Nakai-Sario [1] also proved that $C_{0}=C \in \mathcal{O}_{H^{2} D}$ and $\boldsymbol{C}_{\alpha} \notin \mathcal{O}_{H^{2} D}$ if $\alpha$ is chosen large enough. Again its significance is revealed in an interesting contrast with the harmonic case: $\boldsymbol{C}_{\alpha} \in \mathcal{O}_{H D}$ for every $\alpha$. Although the existence of $\alpha$ with $C_{\alpha} \notin \mathcal{O}_{H^{2} D}$ was assured in [1], its exact determination, which may be useful for e.g. producing a more delicate examples, was left unsettled. Therefore the main object of this paper is to establish a counterpart of the above Theorem 1:

THEOREM 2. The manifold $\boldsymbol{C}_{\alpha}$ belongs to the null class $\mathcal{O}_{H^{2} D}$ if and onlg if $\alpha \leq 3 / 2$.

2. We denote by $\Delta_{\alpha}, d v_{\alpha}$, and $\operatorname{grad}_{\alpha}$ the Laplace-Beltrami operator, the volume element, and the gradient with respect to the Riemannian manifold $\boldsymbol{C}_{\alpha}$. Let $\Delta, d v, \mathrm{grad}$, and $\boldsymbol{C}$ stand for the case $\alpha=0$. By using $\lambda_{\alpha}(z)=(1+|z|)^{-\alpha}$, we see that $\Delta_{\alpha}=\lambda_{\alpha}^{-2} \Delta, d v_{\alpha}=\lambda_{\alpha}^{2} d v$, and $\operatorname{grad}_{\alpha}=$ $\lambda_{\alpha}^{-2}$ grad. Therefore $H\left(C_{\alpha}\right)=H(C), D\left(C_{\alpha}\right)=D(C)$, and $D_{C_{\alpha}}(\varphi)=D_{C}(\varphi)$. A fortiori the assertion $C_{\alpha} \notin \mathcal{O}_{H^{2} D}$ is equivalent to the Poisson equation

$$
\Delta u(z)=\lambda_{\alpha}(z)^{2} h(z)
$$

having a nonharmonic (Euclidean) Dirichlet finite solution $u$ on $C$ for some harmonic function $h$. We denote by $H_{\alpha}(C)$ the class of such harmonic functions. Clearly the constant function 0 does not belong to $H_{\alpha}(\boldsymbol{C})$ but $H_{\alpha}(\boldsymbol{C}) \cup\{0\}$ forms a vector space.

In order to prove Theorem 2, we only have to show that $H_{\alpha}(C)=\emptyset$ if and only if $\alpha \leq 3 / 2$. It will be convenient to provide a test for an $h \in H(C)$ to belong to $H_{\alpha}(C)$. We denote by $(f, g)_{\alpha}$ the inner product of $f$ and $g$ in $L^{2}\left(C_{\alpha}\right)=L^{2}\left(C, \lambda_{\alpha}^{2} d v\right)$ and by $(f, g)$ the $(f, g)_{0}$. Then we have (Nakai-Sario [1])

LEMma 1. A nonzero harmonic function $h$ on $C$ belongs to the class $H_{\alpha}(\boldsymbol{C})$ if and only if

$$
\sup _{\varphi \in C_{0}^{1}(C)}\left|(h, \varphi)_{\alpha}\right|^{2} / D_{C}(\varphi)<\infty .
$$

Here $C_{0}^{1}$ is the class of $C^{1}$-functions with compact supports. To prove Lemma 1 suppose $h \in H_{\alpha}(\boldsymbol{C})$, i.e. (2) has a solution $u \in D(\boldsymbol{C})$. For 
$\varphi \in C_{0}^{1}(C)$, the Green formula yields $(h, \varphi)_{\alpha}=(\Delta u, \varphi)=-D_{C}(u, \varphi)$. By the Schwarz inequality, $\left|(h, \varphi)_{\alpha}\right|^{2} \leq D_{c}(u) \cdot D_{c}(\varphi)$. Conversely suppose (3) is valid. Let $\mathscr{L}$ be the closure of $C_{0}^{\infty}(C)$ in $D(C)$ with respect to $D_{C}(\cdot)$. By the Riesz theorem, there exists $u \in \mathscr{L}$ such that $\ell(\varphi)=D_{C}(u, \varphi)$ for every $\varphi \in \mathscr{L}$ and in particular for every $\varphi \in C_{0}^{\infty}$, where $\ell$ is the bounded extension to $\mathscr{L}$ of $(h, \cdot)_{\alpha}$. Namely, $(h, \varphi)_{\alpha}=-D_{\boldsymbol{c}}(u, \varphi)$ for every $\varphi \in C_{0}^{\infty}(\boldsymbol{C})$. By the Weyl lemma $u$ is a genuine solution of (1) and also $u \in D(C)$.

3. Expand an $h \in H(C)$ into its Fourier series:

$$
h\left(r e^{i \theta}\right)=\sum_{n=0}^{\infty} r^{n}\left(a_{n} \cos n \theta+b_{n} \sin n \theta\right), \quad b_{0}=0
$$

for $r \in[0, \infty)$ and $\theta \in \boldsymbol{R}$. For the sake of simplicity we call $m(h)=$ $\sup \left\{n ; a_{n}^{2}+b_{n}^{2} \neq 0\right\} \leq \infty$ the order of $h$. We denote by $E_{k}$ the class $\{h \in H(C) ; m(h) \leq k\}$ for $k=0,1,2, \cdots$ and we set $E_{k}=\{0\}$ for $k=-1$, $-2, \cdots, E_{k}^{\prime}=\left\{h \in E_{k} ; h \neq \equiv, a_{0}=b_{0}=0\right\}$, for $k=1,2, \cdots$, and $E_{k}^{\prime}=\emptyset$ for $k=0,-1,-2, \cdots$. We first prove

LEMMA 2. If $2 \alpha>k+2 \geq 3$, then $E_{k}^{\prime} \subset H_{\alpha}(C)$.

We only have to show that $r^{n} \cos n \theta$ and $r^{n} \sin n \theta$ belong to $H_{\alpha}(\boldsymbol{C})$ for every $n$ with $1 \leq n<2 \alpha-2$. Since the reasoning is the same, we only show that $r^{n} \cos n \theta \in H_{\alpha}(\boldsymbol{C})$. Let $\varphi \in C_{0}^{\infty}(\boldsymbol{C})$, and expand it into its Fourier series:

$$
\varphi\left(r e^{i \theta}\right)=\sum_{n=0}^{\infty}\left(a_{n}(r) \cos n \theta+b_{n}(r) \sin n \theta\right)
$$

where $a_{n}(r)$ and $b_{n}(r)$ are all in $C_{0}^{\infty}[0, \infty)$. Observe that

$$
D_{C}(\varphi)=\sum \pi\left(\int_{0}^{\infty}\left(a_{n}^{\prime}(r)^{2}+b_{n}^{\prime}(r)^{2}\right) r d r+n^{2} \int_{0}^{\infty}\left(a_{n}(r)^{2}+b_{n}(r)^{2}\right) \frac{d r}{r}\right) .
$$

On the other hand we have

$$
\begin{aligned}
(h, \varphi)_{\alpha} & =\int_{0}^{\infty}\left(\int_{0}^{2 \pi} \varphi\left(r e^{i \theta}\right) \cos n \theta d \theta\right) r^{n+1}(1+r)^{-2 \alpha} d r \\
& =\pi \int_{0}^{\infty} a_{n}(r) r^{n+1}(1+r)^{-2 \alpha} d r .
\end{aligned}
$$

By the Schwarz inequality

$$
\left|(h, \varphi)_{\alpha}\right|^{2} \leq \pi^{2} K_{\alpha} \cdot \int_{0}^{\infty} a_{n}(r)^{2} \frac{d r}{r}
$$


where $K_{\alpha}=\int_{0}^{\infty} r^{2 n+3}(1+r)^{-4 \alpha} d r$ is finite if and only if $2 \alpha>n+2 \geq 3$. By (6) and (7), we have (3) and $r^{n} \cos n \theta \in H_{\alpha}(C)$.

LEMMA 3. If $k+3 \geq 2 \alpha$, then $H_{\alpha}(C) \subset E_{k}^{\prime}$.

Let the Fourier expansion of $h \in H_{\alpha}(C)$ be given by (4) and suppose $a_{n}^{2}+b_{n}^{2} \neq 0$. For $t>1$ let

$$
\rho_{t}(r)= \begin{cases}\left(r-t^{1 / 2}\right)^{2}(t-r)^{2}, & r \in\left[t^{1 / 2}, t\right] ; \\ 0, & r \in[0, \infty)-\left[t^{1 / 2}, t\right],\end{cases}
$$

which belongs to $C_{0}^{1}(0, \infty)$. Then the function

$$
\varphi_{t}\left(r e^{i \theta}\right)=\rho_{t}(r)\left(a_{n} \cos n \theta+b_{n} \sin n \theta\right)
$$

belongs to $C_{0}^{1}(C)$. By an easy computation we find the universal positive constants $A, B$ and $t_{0}>1$ such that

$$
\left(h, \tau \varphi_{t}\right)_{\alpha} \geq A \tau t^{6+n-2 \alpha}, \quad D_{c}\left(\tau \varphi_{t}\right) \leq B \tau^{2} t^{8}
$$

for every $t>t_{0}$ and $\tau>0$. If $6+n-2 \alpha>4$, then (9) implies that $\left|\left(h, \varphi_{t}\right)_{\alpha}\right|^{2} / D_{c}\left(\varphi_{t}\right) \rightarrow \infty$, which contradicts (3). If $6+n-2 \alpha=4$, then (9) takes the form

$$
\left(h, \tau \varphi_{t}\right)_{\alpha} \geq A \tau t^{4}, \quad D_{C}\left(\tau \varphi_{t}\right) \leq B \tau^{2} t^{8} .
$$

Let $\left\{t_{\nu}\right\}_{\nu=0}^{\infty}$ be a sequence of real numbers such that $t_{\nu}+\nu<t_{\nu+1}^{1 / 2}$. Next consider a sequence $\left\{\tau_{\nu}\right\}_{\nu=1}^{\infty}$ given by

$$
\tau_{\nu} t_{\nu}^{4}=\nu^{-1} \quad(\nu=1,2, \cdots) .
$$

We then consider a sequence $\left\{\Phi_{\mu}\right\}_{\mu=1}^{\infty}$ of functions $\Phi_{\mu}$ in $C_{0}^{1}(\boldsymbol{C})$ given by

$$
\Phi_{\mu}\left(r e^{i \theta}\right)=\sum_{\nu=1}^{\mu} \tau_{\nu} \varphi_{t_{\nu}}\left(r e^{i \theta}\right)
$$

By (10) and (11) we deduce that

$$
\left(h, \Phi_{\mu}\right)_{\alpha} \geq A \sum_{\nu=1}^{\mu} \nu^{-1}
$$

By definition (12) we see that $\left(\partial \Phi_{\mu} / \partial x^{i}\right)^{2}=\sum_{\nu=1}^{\mu}\left(\tau_{\nu} \partial \varphi_{\nu} / \partial x^{i}\right)^{2}(i=1,2)$, and a fortiori $D_{C}\left(\Phi_{\mu}\right)=\sum_{\nu=1}^{\mu} D_{c}\left(\tau_{\nu} \varphi_{\nu}\right)$. Again by (10) and (11) we obtain

$$
D_{C}\left(\Phi_{\mu}\right) \leq B \sum_{\nu=1}^{\mu} \nu^{-2}
$$

From (13) and (14) it follows that 


$$
\left|\left(h, \Phi_{\mu}\right)_{\alpha}\right|^{2} / D_{C}\left(\Phi_{\mu}\right) \geq\left(A^{2} / B\right)\left(\sum_{\nu=1}^{\mu} \nu^{-1}\right)^{2} /\left(\sum_{\nu=1}^{\mu} \nu^{-2}\right) \rightarrow \infty
$$

as $\mu \rightarrow \infty$, in violation of (3). Hence $n$ must satisfy $6+n-2 \alpha<4$, i.e. $n+2<2 \alpha \leq k+3$. Then $n \leq k$, and $H_{\alpha}(C) \subset E_{k}$. Because of Lemma 2

$$
a_{0}=h\left(r e^{i \theta}\right)-\sum_{n=1}^{m(h)} r^{n}\left(a_{n} \cos n \theta+b_{n} \sin n \theta\right)
$$

must belong to $H_{a}(C)$ unless $a_{0}=0$. It is easy to find a bounded sequence $\left\{\varphi_{\mu}\right\}_{1}^{\infty} \subset C_{0}^{1}(C)$ such that $\varphi_{\mu}$ converges to 1 and $D_{C}\left(\varphi_{\mu}\right) \rightarrow 0$. If $a_{0} \in H_{\alpha}(C)$, then $\left|\left(a_{0}, \varphi_{\mu}\right)\right|^{2} \rightarrow\left(2 \pi a_{0} \int_{0}^{\infty}(1+r)^{-2 \alpha} r d r\right)^{2}>0$; but $D_{C}\left(\varphi_{\mu}\right) \rightarrow 0$ as $\mu \rightarrow \infty$, in violation of (3). Therefore $\alpha_{0}=0$ and $h \in E_{k}^{\prime}$, i.e. $H_{\alpha}(C) \subset E_{k}^{\prime}$.

4. Suppose that $H_{\alpha}(C)=\emptyset$. If $2 \alpha>1+2=3$, then by Lemma 2 , $E_{1}^{\prime} \subset H_{\alpha}(C)$, a contradiction. Therefore $2 \alpha \leq 3$. Conversely suppose that $2 \alpha \leq 3$, i.e. $0+3 \geq 2 \alpha$. By Lemma 3 we see that $H_{\alpha}(C) \subset E_{0}^{\prime}=\emptyset$. Thus $H_{\alpha}(C)=\emptyset$ if and only if $\alpha \leq 3 / 2$. This completes the proof of Theorem 2 .

5. Let $u_{1}$ and $u_{2}$ be Dirichlet finite solutions of (2). Then $u_{1}-u_{2}$ is a Dirichlet finite harmonic function on $\boldsymbol{C}$, i.e. $u_{1}-u_{2} \in H D(\boldsymbol{C})=\boldsymbol{R}$. Therefore the vector space $H^{2} D\left(\boldsymbol{C}_{\alpha}\right) / \boldsymbol{R}$ is isomorphic to $H_{\alpha}(\boldsymbol{C}) \cup\{0\}$. By Lemmas 2 and $3, H_{\alpha}(C) \cup\{0\}=E_{k}^{\prime}(2 \alpha-2>k \geq 2 \alpha-3)$. Since $\operatorname{dim} E_{k}^{\prime}$ $=2 k$ for $k>0$ and $=0$ for $k \leq 0$, as a more precise form of Theorem 2 , we obtain

THEOREM 3. Let $d_{\alpha}$ be the dimension of the vector space $H^{2} D\left(C_{\alpha}\right) /$ $H D\left(C_{\alpha}\right)=H^{2} D\left(C_{\alpha}\right) / R$. If $\alpha \leq 3 / 2$, then $d_{\alpha}=0$. If $\alpha>3 / 2$, then $d_{\alpha}=2 k_{\alpha}$ with $2 \alpha-2>k_{\alpha} \geq 2 \alpha-3$.

\section{REFERENCES}

[1] M. Nakai-L. Sario: Existence of Dirichlet finite biharmonic functions, Ann. Acad. Sci. Fenn., 532 (1973), 1-34.

[2] H. O'Malla: Dirichlet finite biharmonic functions on the unit disk with distorted metrics, Proc. Amer. Math. Soc., 32 (1972), 521-524.

[ 3 ] L. Sario-M. Nakai: Classification Theory of Riemann Surfaces, Springer, 1970.

Nagoya University 